\title{
Missing Maps Project and Its Use in Education
}

\author{
Jan Brodský \\ Faculty of Science, Masaryk University, Brno, Jan Brodský - broda@ mail.muni.cz
}

Keywords: Missing Maps, OpenStreetMap, Doctors Without Borders, Volunteered Geographic Information, remote sensing, education, mapathon, crisis management

\begin{abstract}
:
The article is about OpenStreetMap and Missing Maps Project and their introducing in the remote sensing classes at universities to show potential of geographic information to improve education of people about how to help to solve the disaster and crisis situations in the World. This approach also serves as a real model for learning to interpret satellite imagery in such situations.

At the beginning of the article different kinds of disasters are briefly mentioned including their causes and effect on the life of people. Then crisis management is briefly introduced from the point of view selected voluntary projects which are analysed to make process more targeted and efficient. After that Missing Maps project is introduced together with OpenStreetMap and their outputs on the example of non-profit organization Doctors Without Borders (MSF) operating in different parts of the World. In the next part the ways how to contribute and improve their activities and how the whole cooperation works are arranged. Next part of the article focuses on Missing Maps mapathons (meetings of volunteers who create open-source maps for MSF) and the way how they are organized in Brno, Czechia. Editing of the OpenStreetMap for the Missing Maps project has been recently introduced to usual remote sensing classes for students of Masaryk University in Brno. Information about this activity is also described.
\end{abstract}

